CRYSTALLOGRAPHIC COMMUNICATIONS

ISSN 2056-9890

Received 17 May 2018

Accepted 1 June 2018

Edited by C. Rizzoli, Universita degli Studi di Parma, Italy

Keywords: crystal structure; piperidine-4-one Mannich reaction; Hirshfeld surface analysis.

CCDC reference: 1846705

Supporting information: this article has supporting information at journals.iucr.org/e

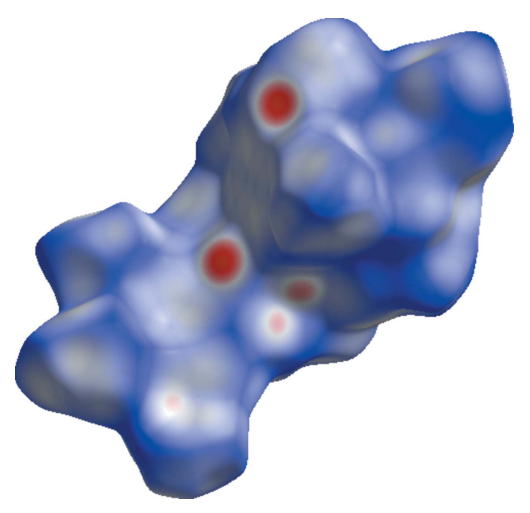

OPEN $\odot$ ACCESS

\section{Crystal structure and Hirshfeld surface analysis of 3-octyl-4-oxo-2,6-bis(3,4,5-trimethoxyphenyl)- piperidinium chloride}

Rubina Siddiqui, ${ }^{a}$ Urooj Iqbal, ${ }^{\text {b }}$ Zafar Saeed Saify, ${ }^{\text {a }}$ Shammim Akhter ${ }^{\mathrm{a}}$ and Sammer Yousuf $^{\mathrm{b}_{*}}$

a Department of Pharmaceutical Chemistry, Faculty of Pharmacy and Pharmaceutical Sciences, University of Karachi, Karachi-75270, Pakistan, and ${ }^{\mathbf{b}} \mathrm{H}$. E. J. Research Institute of Chemistry, International Center for Chemical and Biological Sciences, University of Karachi, Karachi-75270, Pakistan. *Correspondence e-mail: dr.sammer.yousuf@gmail.com

The title compound, $\mathrm{C}_{31} \mathrm{H}_{46} \mathrm{NO}_{7}^{+} \cdot \mathrm{Cl}^{-}$, was synthesized by a one-pot Mannich condensation reaction. In the molecule, the piperidinone ring adopts a chair conformation, and the trimethoxy-substituted benzene rings and octyl chain are arranged equatorially. In the crystal, centrosymmetric dimers are linked into layers parallel to (011) by $\mathrm{N}-\mathrm{H} \cdots \mathrm{Cl}$ and $\mathrm{C}-\mathrm{H} \cdots \mathrm{Cl}$ hydrogen bonds. A Hirshfeld surface analysis indicates that the most important contributions for the crystal packing are $\mathrm{O} \cdots \mathrm{H}(20.5 \%)$ interactions followed by $\mathrm{C} \cdots \mathrm{H}(7.8 \%)$, $\mathrm{Cl} \cdots \mathrm{H}(5.5 \%), \mathrm{C} \cdots \mathrm{C}(1.2 \%), \mathrm{C} \cdots \mathrm{O}(0.5 \%)$ and $\mathrm{Cl} \cdots \mathrm{O}(0.4 \%)$ interactions.

\section{Chemical context}

Piperidine is a naturally occurring bioactive alkaloid (Hu et al., 2002; Finke et al., 2001; Taniguchi \& Ogasawara, 2000) and the heterocyclic six-membered nitrogen-containing piperidine ring is an essential structural part of many important drugs including paroxetine, raloxifene, haloperidol, droperidol and minoxidiln (Wagstaff et al., 2002). 2,6-Diphenyl-substituted piperdine-4-one derivatives are important because of their potential biological activities such as antitumor, antimicrobial, analgesic, local anesthetic, antidepressant and anti-inflammatory (Kálai et al., 2011; Leonova et al., 2010; El-Subbagh et al., 2000; Jerom \& Spencer, 1988). This wide range of biological activities prompted us to synthesize novel 2,6-diphenyl piperdine-4-one derivatives with enhanced biological activities. In a continuation of this work, the title compound was synthesized using a one-pot Mannich condensation reaction as reported by Noller \& Baliah (1948). The adopted one-pot reaction is convenient, simple, easy way for separation of the product with possible high yield. A Hirshfield surface analysis of the title compound was carried out in order to study how different functionalities can affect the crystal packing.<smiles>CCCCCCCCC1C(=O)CC(c2cc(OC)c(OC)c(OC)c2)[NH+]([ClH+])C1c1cc(OC)c(OC)c(OC)c1</smiles> 


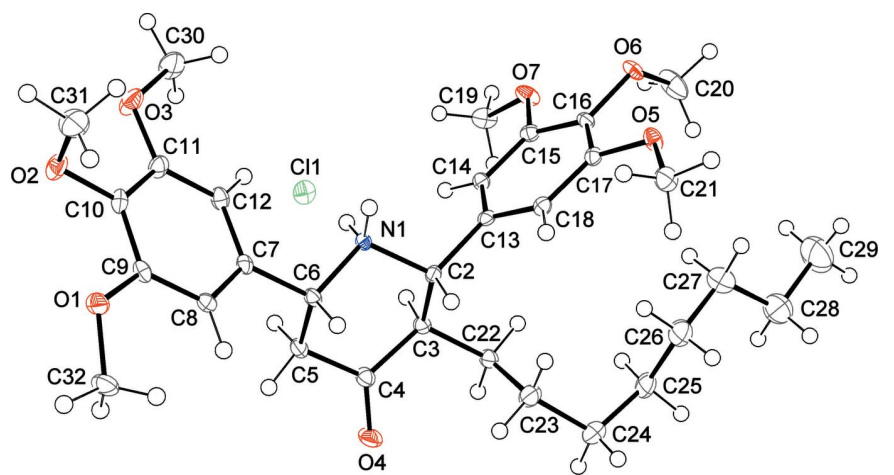

Figure 1

The molecular structure of the title compound with displacement ellipsoids drawn at the $50 \%$ probability level.

\section{Structural commentary}

In the molecule of the title compound (Fig. 1), the heterocyclic six-membered 4-piperidone ring (N1/C2-C6) adopts a chair conformation, with puckering parameters $Q=0.5750$ (15) $\AA, \theta$ $=13.60(14)^{\circ}$ and $\varphi=5.55(61)^{\circ}$. The octyl chain at $\mathrm{C} 3$, and the trimethoxy-substituted benzene rings attached at $\mathrm{C} 2$ and $\mathrm{C} 6$ are equatorially oriented. The trimethoxy benzene rings $\mathrm{C} 7-$ $\mathrm{C} 12$ and $\mathrm{C} 13-\mathrm{C} 18$ form a dihedral angle of $73.91(5)^{\circ}$, and are tilted with respect to the mean plane of the piperidone ring by $59.42(4)$ and $78.54(6)^{\circ}$, respectively. The $\mathrm{C} 13-\mathrm{C} 2-\mathrm{C} 3-\mathrm{C} 22$ and $\mathrm{O} 4-\mathrm{C} 4-\mathrm{C} 3-\mathrm{C} 22$ torsion angles are $56.36(17)$ and $-11.0(2)^{\circ}$, respectively.

\section{Supramolecular features}

In the crystal, centrosymmetrically-related molecules are linked into dimers through pairs of $\mathrm{N}-\mathrm{H} \cdots \mathrm{O}$ hydrogen bonds (Table 1) forming rings with an $R_{2}^{2}(16)$ graph-set motif.
Table 1

Hydrogen-bond geometry $\left(\AA{ }^{\circ}\right)$.

\begin{tabular}{lllll}
\hline$D-\mathrm{H} \cdots A$ & $D-\mathrm{H}$ & $\mathrm{H} \cdots A$ & $D \cdots A$ & $D-\mathrm{H} \cdots A$ \\
\hline $\mathrm{N} 1-\mathrm{H} 1 A \cdots \mathrm{O} 6^{\mathrm{i}}$ & $0.92(2)$ & $1.93(2)$ & $2.8500(18)$ & $175.9(19)$ \\
$\mathrm{N} 1-\mathrm{H} 2 A \cdots \mathrm{Cl} 1$ & $0.92(2)$ & $2.18(2)$ & $3.0959(15)$ & $172.6(19)$ \\
$\mathrm{C} 6-\mathrm{H} 6 \cdots \mathrm{Cl}^{\mathrm{ii}}$ & 1.00 & 2.74 & $3.6526(17)$ & 152 \\
$\mathrm{C} 2-\mathrm{H} 2 \cdots \mathrm{Cl}{ }^{\mathrm{ii}}$ & 1.00 & 2.57 & $3.5153(16)$ & 158 \\
$\mathrm{C} 12-\mathrm{H} 12 \cdots \mathrm{Cl} 1$ & 0.95 & 2.83 & $3.6625(18)$ & 147 \\
$\mathrm{C} 14-\mathrm{H} 14 \cdots \mathrm{Cl} 1$ & 0.95 & 2.82 & $3.6144(16)$ & 141 \\
$\mathrm{C}^{2} 8-\mathrm{H} 28 B \cdots \mathrm{O}{ }^{\mathrm{iii}}$ & 0.99 & 2.52 & $3.308(3)$ & 136 \\
\hline
\end{tabular}

Symmetry codes: $x+1,-y+\frac{1}{2}, z+\frac{1}{2}$.

(i) $-x+1,-y+1,-z+1 ; \quad$ (ii) $\quad x,-y+\frac{1}{2}, z+\frac{1}{2}$;

The dimers are further connected by $\mathrm{N}-\mathrm{H} \cdots \mathrm{Cl}$ and $\mathrm{C}-$ $\mathrm{H} \cdots \mathrm{Cl}$ hydrogen interactions, forming layers parallel to the (011) plane (Fig. 2).

\section{Hirshfeld surface analysis}

A quantitative analysis of all type of interactions in the title compound was performed using Hirshfeld surface analysis. The Hirshfeld surface mapped over $d_{\text {norm }}$ (Spackman \& Jayatilaka, 2009) is shown in Fig. 3 where the red areas on the surface indicate short contacts (as compared to the sum of the van der Waals radii), while the blue areas indicate longer contacts and white areas indicate contacts with distances equal to the sum of the van der Waals radii. Two-dimensional fingerprint plots are shown in Fig. 4 with a broad hump showing $\mathrm{H} \cdots \mathrm{H}$ contacts and intense spikes indicating a strong $\mathrm{O} \cdots \mathrm{H}$ interaction, while the broadening in the wing of the $\mathrm{C} \cdots \mathrm{H}$ interaction is due to the presence of a $\mathrm{Cl} \cdots \mathrm{H}$ interaction The largest contribution is from $\mathrm{H} \cdots \mathrm{H}$ interactions (64.1\%), followed by $\mathrm{O} \cdots \mathrm{H}$ interactions, contributing $20.5 \%$. Other weak intermolecular interactions are: $\mathrm{C} \cdot \mathrm{H}(7.8 \%)$,

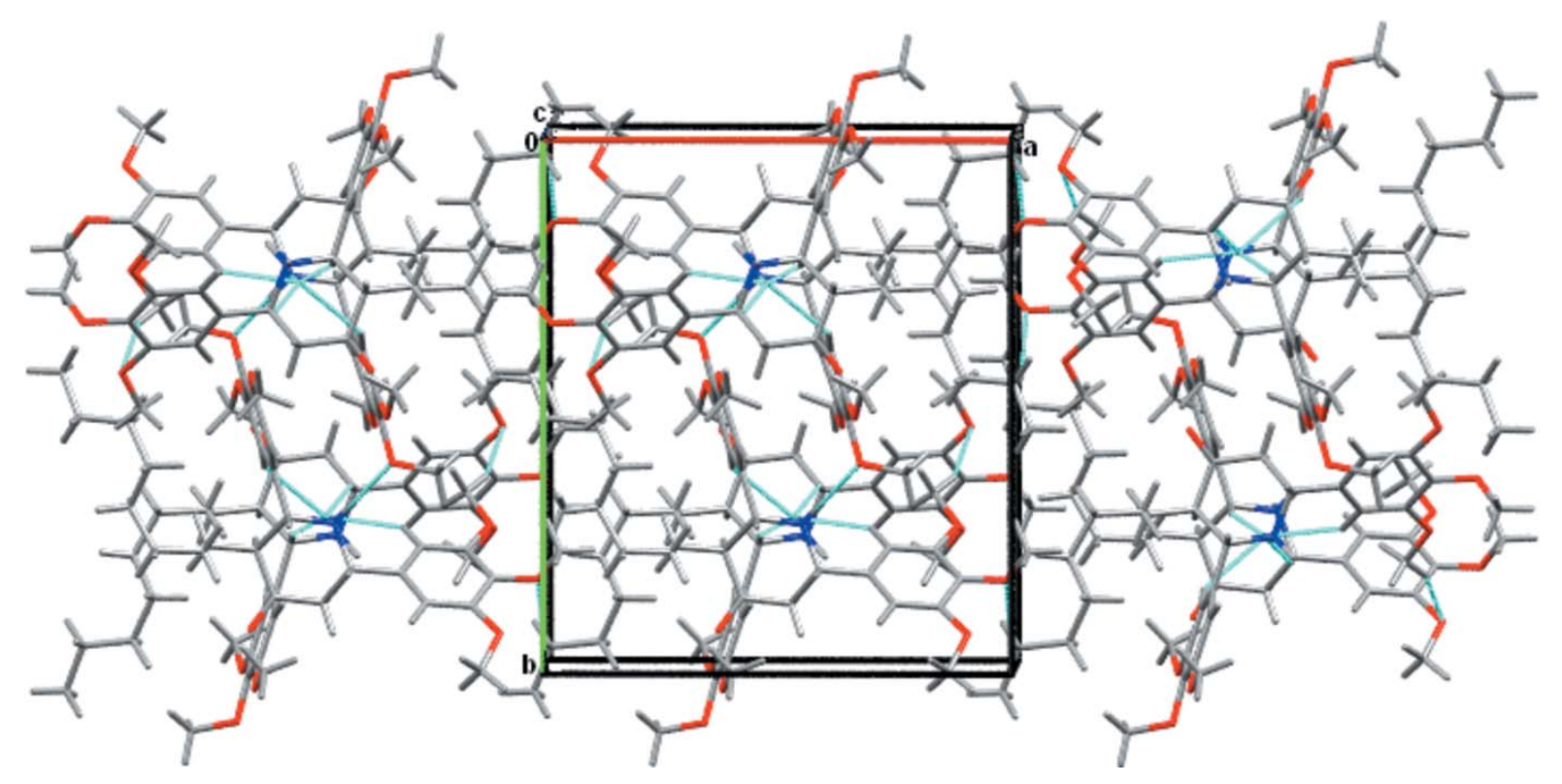

Figure 2

Packing diagram of the title compound viewed approximately along the $c$ axis. Turquoise lines indicate hydrogen bonds. 


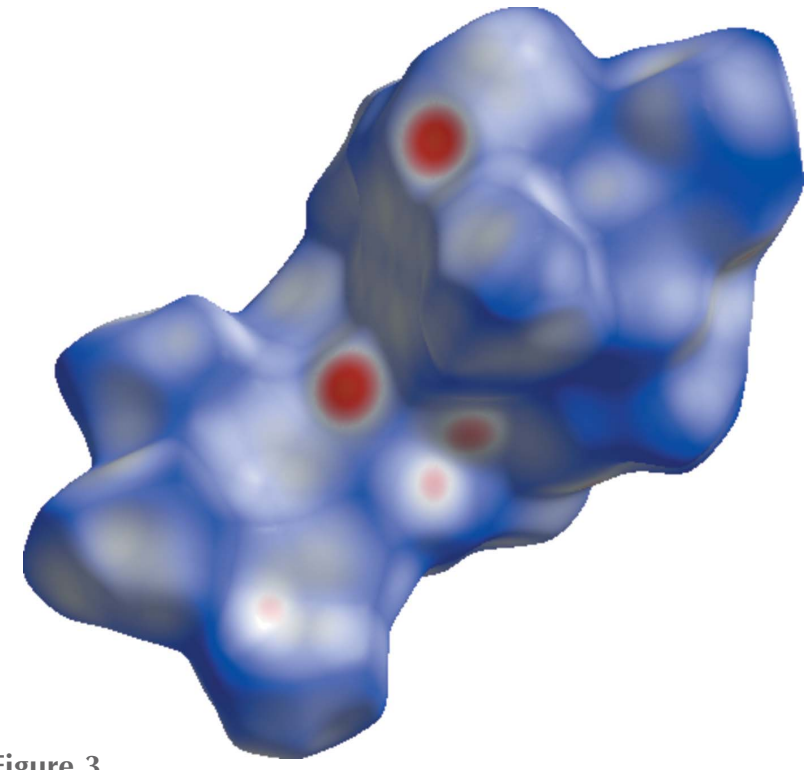

Figure 3

Hirshfeld surface mapped ove $d_{\text {norm }}$ showing the intermolecular contacts in the title compound.

$\mathrm{Cl} \cdots \mathrm{H}(5.5 \%), \mathrm{C} \cdots \mathrm{C}(1.2 \%), \mathrm{C} \cdots \mathrm{O}(0.5 \%)$ and $\mathrm{Cl} \cdots \mathrm{O}$ $(0.4 \%)$.

\section{Database survey}

A search of the Cambridge Crystallographic Database (CSD version 5.39, updates February 2018; Groom et al., 2016) revealed three examples of organic compounds having piperdine-4-one as the central unit, namely 1-acryloyl-3-methyl-2,6bis(3,4,5-trimethoxyphenyl)piperidine-4-one (Gnanendra et al., 2009), $N$-nitroso-2,6-di(3,4,5-trimethoxyphenyl)-3,5-dimethylpiperidin-4-one (Kumaran, et al., 1999) and 1-(2chloroacetyl)-3-methyl-2,6-bis(3,4,5-trimethoxyphenyl)piper- idine-4-one (Lakshminarayana et al., 2009). A study of the supramolecular features of these compounds revealed that the crystal lattices are stabilized mainly by $\mathrm{C}-\mathrm{H} \cdots \mathrm{O}$ intermolecular interactions, forming two-dimensional networks.

\section{Synthesis and crystallization}

The title compound was synthesized according to the procedure given in literature (Noller \& Baliah, 1948). A mixture of 2-undecanone, $\quad(0.206 \mathrm{ml}, 1 \mathrm{mmol}), \quad 3,4,5$-trimethoxybenzaldehyde $(0.39 \mathrm{~g}, 2 \mathrm{mmol})$ and ammonium acetate $(0.077 \mathrm{~g}, 1 \mathrm{mmol})$ in ethanol $(50 \mathrm{ml})$ was allowed to reflux for three hours. The progress of reaction was monitored by TLC. After completion of the reaction, the mixture was acidified with dilute hydrochloric acid $(5 \mathrm{~mL})$ and the resulting precipitate was collected, washed with an ethanol-ether mixture $(1: 4 v / v)$, dried and redissolved in ethanol. Crystals suitable for single-crystal X-ray diffraction analysis were obtained on slow evaporation of the solvent at room temperature.

\section{Refinement}

Crystal data, data collection and structure refinement details are summarized in Table $2 . \mathrm{H}$ atoms on methyl, methylene and benzene were positioned geometrically with $\mathrm{C}-\mathrm{H}=0.95-$ $1.00 \AA$ and constrained to ride on their parent atoms with $U_{\text {iso }}(\mathrm{H})=1.2 U_{\text {eq }}(\mathrm{C})$ or $1.5 U_{\text {eq }}(\mathrm{C})$ for methyl $\mathrm{H}$ atoms. A rotating model was used for the methyl groups. The N-bound hydrogen atoms were located in a difference-Fourier map and freely refined.

\section{Funding information}

The authors acknowledge the financial support of the Higher Education Commission of Pakistan (HEC) through research
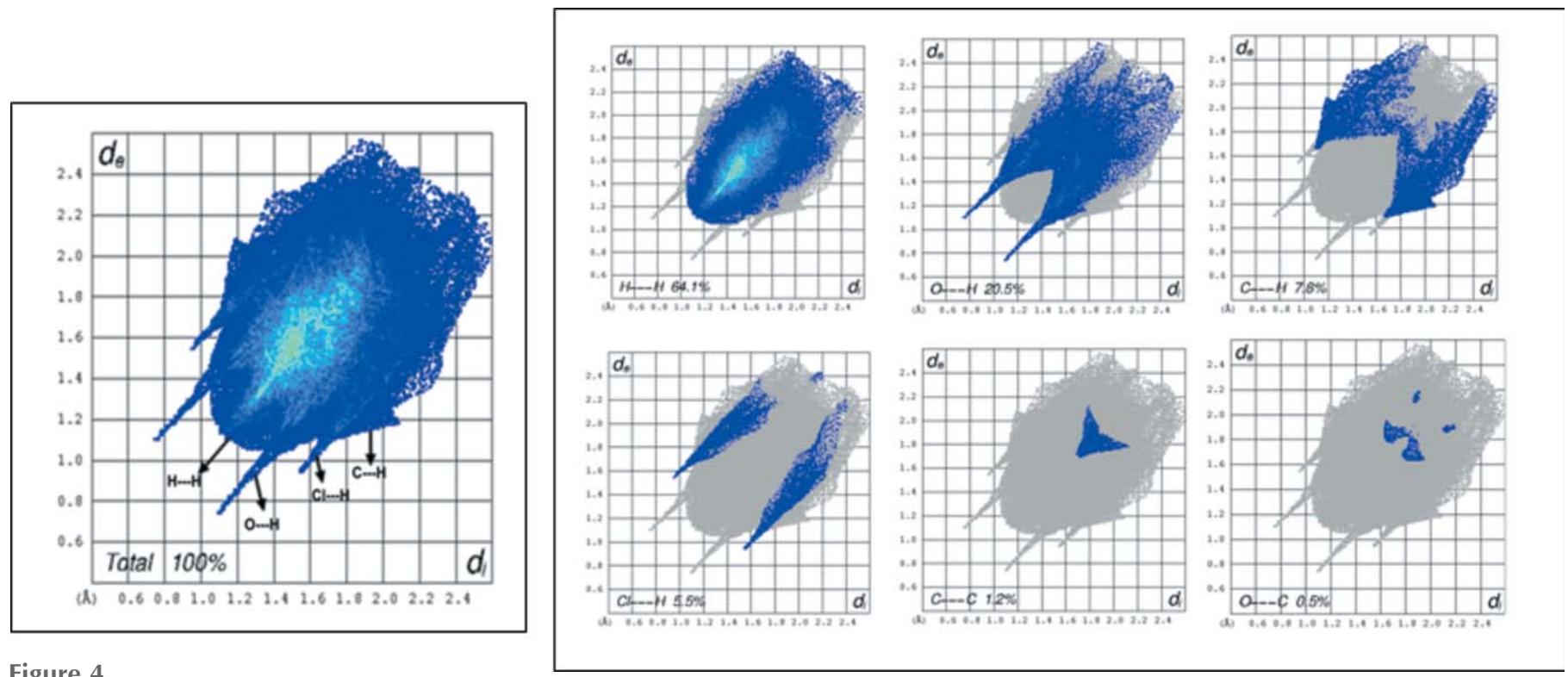

Figure 4

Two-dimensional fingerprint plots for the title compound. 
Table 2

Experimental details.

\begin{tabular}{|c|c|}
\hline \multicolumn{2}{|l|}{ Crystal data } \\
\hline Chemical formula & $\mathrm{C}_{31} \mathrm{H}_{46} \mathrm{NO}_{7}^{+} \cdot \mathrm{Cl}^{-}$ \\
\hline$M_{\mathrm{r}}$ & 580.14 \\
\hline Crystal system, space group & Monoclinic, $P 2_{1} / c$ \\
\hline Temperature (K) & 100 \\
\hline$a, b, c(\AA)$ & $\begin{array}{l}14.1073(3), 16.0156(3), \\
13.7785(3)\end{array}$ \\
\hline$\beta\left({ }^{\circ}\right)$ & $95.006(1)$ \\
\hline$V\left(\AA^{3}\right)$ & $3101.20(11)$ \\
\hline$Z$ & 4 \\
\hline Radiation type & $\mathrm{Cu} K \alpha$ \\
\hline$\mu\left(\mathrm{mm}^{-1}\right)$ & 1.47 \\
\hline Crystal size $(\mathrm{mm})$ & $0.20 \times 0.13 \times 0.06$ \\
\hline \multicolumn{2}{|l|}{ Data collection } \\
\hline Diffractometer & Bruker APEXII CCD \\
\hline Absorption correction & $\begin{array}{l}\text { Multi-scan (SADABS; Bruker, } \\
\text { 2014) }\end{array}$ \\
\hline$T_{\min }, T_{\max }$ & $0.758,0.917$ \\
\hline $\begin{array}{l}\text { No. of measured, independent and } \\
\text { observed }[I>2 \sigma(I)] \text { reflections }\end{array}$ & $42615,5681,4654$ \\
\hline$R_{\text {int }}$ & 0.070 \\
\hline$(\sin \theta / \lambda)_{\max }\left(\AA^{-1}\right)$ & 0.602 \\
\hline \multicolumn{2}{|l|}{ Refinement } \\
\hline$R\left[F^{2}>2 \sigma\left(F^{2}\right)\right], w R\left(F^{2}\right), S$ & $0.038,0.094,1.01$ \\
\hline No. of reflections & 5681 \\
\hline No. of parameters & 376 \\
\hline $\mathrm{H}$-atom treatment & $\begin{array}{l}\mathrm{H} \text { atoms treated by a mixture of } \\
\text { independent and constrained } \\
\text { refinement }\end{array}$ \\
\hline$\Delta \rho_{\max }, \Delta \rho_{\min }\left(\mathrm{e} \AA^{-3}\right)$ & $0.30,-0.26$ \\
\hline
\end{tabular}

Computer programs: APEX3 and SAINT (Bruker, 2014), SHELXT (Sheldrick, 2015a), SHELXL2016 (Sheldrick, 2015b) and SHELXTL (Sheldrick, 2008).

project No. 20-2830 under the National Research Program for Universities.

\section{References}

Bruker (2014). APEX3, SAINT and SADABS. Bruker ASX Inc., Madison, Wisconsin, USA

El-Subbagh, H. I., Abu-Zaid, S. M., Mahran, M. A., Badria, F. A. \& Al-Obaid, A. M. (2000). J. Med. Chem. 43, 2915-2921.

Finke, P. E., Oates, B., Mills, S. G., MacCoss, M., Malkowitz, L., Springer, M. S., Gould, S. L., DeMartino, J. A., Carella, A., Carver, G., Holmes, K., Danzeisen, R., Hazuda, D., Kessler, J., Lineberger, J., Miller, M., Schleif, W. A. \& Emini, E. A. (2001). Bioorg. Med. Chem. Lett. 11, 2475-2479.

Gnanendra, C. R., Lakshminarayana, B. N., Thippeswamy, G. B., Sridhar, M. A., Naik, N. \& Prasad, J. S. (2009). Mol. Cryst. Liq. Cryst. 515, 179-189.

Groom, C. R., Bruno, I. J., Lightfoot, M. P. \& Ward, S. C. (2016). Acta Cryst. B72, 171-179.

Hu, X. E., Kim, N. K. \& Ledoussal, B. (2002). Org. Lett. 4, 4499-4502. Jerom, B. R. \& Spencer, K. H. (1988). European Patent Application EP277794.

Kálai, T., Kuppusamy, M. L., Balog, M., Selvendiran, K., Rivera, B. K., Kuppusamy, P. \& Hideg, K. (2011). J. Med. Chem. 54, 5414-5421.

Kumaran, D., Ponnuswamy, M. N., Shanmugam, G., Thenmozhiyal, J. C., Jeyaraman, R., Panneerselvam, K. \& Soriano-Garcia, M. (1999). J. Chem. Cryst. 29, 769-775.

Lakshminarayana, B. N., Shashidhara Prasad, J., Gnanendra, C. R., Sridhar, M. A. \& Chenne Gowda, D. (2009). Acta Cryst. E65, o1237.

Leonova, E., Makarov, M., Klemenkova, Z. \& Odinets, I. (2010). Helv. Chim. Acta, 93, 1990-1999.

Noller, C. R. \& Baliah, V. (1948). J. Am. Chem. Soc. 70, 3853-3855.

Sheldrick, G. M. (2008). Acta Cryst. A64, 112-122.

Sheldrick, G. M. (2015a). Acta Cryst. A71, 3-8.

Sheldrick, G. M. (2015b). Acta Cryst. C71, 3-8.

Spackman, M. A. \& Jayatilaka, D. (2009). CrystEngComm, 11, 19-32. Taniguchi, T. \& Ogasawara, K. (2000). Org. Lett. 2, 3193-3195.

Wagstaff, A. J., Cheer, S. M., Matheson, A. J., Ormrod, D. \& Goa, K. L. (2002). Drugs, 62, 655-703. 


\section{supporting information}

Acta Cryst. (2018). E74, 931-934 [https://doi.org/10.1107/S2056989018008125]

Crystal structure and Hirshfeld surface analysis of 3-octyl-4-oxo-2,6-bis(3,4,5trimethoxyphenyl)piperidinium chloride

Rubina Siddiqui, Urooj Iqbal, Zafar Saeed Saify, Shammim Akhter and Sammer Yousuf

Computing details

Data collection: APEX3 (Bruker, 2014); cell refinement: SAINT (Bruker, 2014); data reduction: SAINT (Bruker, 2014); program(s) used to solve structure: SHELXT (Sheldrick, 2015a); program(s) used to refine structure: SHELXL2016 (Sheldrick, 2015b); molecular graphics: SHELXTL (Sheldrick, 2008); software used to prepare material for publication: SHELXTL (Sheldrick, 2008).

3-Octyl-4-oxo-2,6-bis(3,4,5-trimethoxyphenyl)piperidinium chloride

Crystal data

$\mathrm{C}_{31} \mathrm{H}_{46} \mathrm{NO}_{7}^{+} \cdot \mathrm{Cl}^{-}$

$M_{r}=580.14$

Monoclinic, $P 2_{1} / c$

$a=14.1073$ (3) $\AA$

$b=16.0156(3) \AA$

$c=13.7785$ (3) $\AA$

$\beta=95.006(1)^{\circ}$

$V=3101.20(11) \AA^{3}$

$Z=4$

\section{Data collection}

Bruker APEXII CCD diffractometer

$\varphi$ and $\omega$ scans

Absorption correction: multi-scan

(SADABS; Bruker, 2014)

$T_{\min }=0.758, T_{\max }=0.917$

42615 measured reflections

\section{Refinement}

Refinement on $F^{2}$

Least-squares matrix: full

$R\left[F^{2}>2 \sigma\left(F^{2}\right)\right]=0.038$

$w R\left(F^{2}\right)=0.094$

$S=1.01$

5681 reflections

376 parameters

0 restraints
$F(000)=1248$

$D_{\mathrm{x}}=1.243 \mathrm{Mg} \mathrm{m}^{-3}$

$\mathrm{Cu} K \alpha$ radiation, $\lambda=1.54178 \AA$

Cell parameters from 9910 reflections

$\theta=4.2-68.1^{\circ}$

$\mu=1.47 \mathrm{~mm}^{-1}$

$T=100 \mathrm{~K}$

Plate, colourless

$0.20 \times 0.13 \times 0.06 \mathrm{~mm}$

5681 independent reflections

4654 reflections with $I>2 \sigma(I)$

$R_{\text {int }}=0.070$

$\theta_{\max }=68.2^{\circ}, \theta_{\min }=3.1^{\circ}$

$h=-16 \rightarrow 16$

$k=-19 \rightarrow 19$

$l=-16 \rightarrow 16$

Hydrogen site location: mixed

$\mathrm{H}$ atoms treated by a mixture of independent and constrained refinement

$w=1 /\left[\sigma^{2}\left(F_{\mathrm{o}}{ }^{2}\right)+(0.0429 P)^{2}+1.6956 P\right]$ where $P=\left(F_{\mathrm{o}}^{2}+2 F_{\mathrm{c}}^{2}\right) / 3$

$(\Delta / \sigma)_{\max }<0.001$

$\Delta \rho_{\max }=0.30$ e $\AA^{-3}$

$\Delta \rho_{\min }=-0.25$ e $\AA^{-3}$ 


\section{Special details}

Geometry. All esds (except the esd in the dihedral angle between two 1.s. planes) are estimated using the full covariance matrix. The cell esds are taken into account individually in the estimation of esds in distances, angles and torsion angles; correlations between esds in cell parameters are only used when they are defined by crystal symmetry. An approximate (isotropic) treatment of cell esds is used for estimating esds involving l.s. planes.

Fractional atomic coordinates and isotropic or equivalent isotropic displacement parameters $\left(\AA^{2}\right)$

\begin{tabular}{|c|c|c|c|c|}
\hline & $x$ & $y$ & $z$ & $U_{\text {iso }} * / U_{\text {eq }}$ \\
\hline $\mathrm{C} 11$ & $0.47703(3)$ & $0.27339(3)$ & $0.21759(3)$ & $0.01915(11)$ \\
\hline N1 & $0.45438(9)$ & $0.26752(8)$ & $0.43896(10)$ & $0.0101(3)$ \\
\hline H1A & $0.4132(15)$ & $0.3058(13)$ & $0.4627(15)$ & $0.027(6)^{*}$ \\
\hline $\mathrm{H} 2 \mathrm{~A}$ & $0.4593(14)$ & $0.2742(12)$ & $0.3734(16)$ & $0.022(5)^{*}$ \\
\hline $\mathrm{O} 1$ & $0.10007(8)$ & $0.06252(7)$ & $0.49865(9)$ & $0.0175(3)$ \\
\hline $\mathrm{O} 2$ & $0.02051(8)$ & $0.16016(8)$ & $0.35747(9)$ & $0.0204(3)$ \\
\hline $\mathrm{O} 3$ & $0.12362(9)$ & $0.26030(8)$ & $0.25424(9)$ & $0.0254(3)$ \\
\hline $\mathrm{O} 4$ & $0.63342(9)$ & $0.06866(7)$ & $0.46982(10)$ & $0.0244(3)$ \\
\hline O5 & $0.62986(8)$ & $0.55158(7)$ & $0.64533(8)$ & $0.0162(3)$ \\
\hline O6 & $0.67065(8)$ & $0.61776(7)$ & $0.47872(8)$ & $0.0149(3)$ \\
\hline $\mathrm{O} 7$ & $0.66126(9)$ & $0.52733(7)$ & $0.31193(8)$ & $0.0185(3)$ \\
\hline $\mathrm{C} 12$ & $0.27045(12)$ & $0.22067(10)$ & $0.34915(12)$ & $0.0148(3)$ \\
\hline H12 & 0.308243 & 0.255742 & 0.312448 & $0.018^{*}$ \\
\hline $\mathrm{C} 11$ & $0.17271(12)$ & $0.21537(11)$ & $0.32608(12)$ & $0.0162(4)$ \\
\hline $\mathrm{C} 10$ & $0.11760(11)$ & $0.16272(11)$ & $0.37882(12)$ & $0.0147(3)$ \\
\hline C9 & $0.16088(12)$ & $0.11261(10)$ & $0.45268(12)$ & $0.0132(3)$ \\
\hline $\mathrm{C} 8$ & $0.25830(12)$ & $0.11864(10)$ & $0.47765(12)$ & $0.0127(3)$ \\
\hline $\mathrm{H} 8$ & 0.287831 & 0.085463 & 0.528866 & $0.015^{*}$ \\
\hline $\mathrm{C} 7$ & $0.31232(11)$ & $0.17396(10)$ & $0.42668(11)$ & $0.0117(3)$ \\
\hline C6 & $0.41701(11)$ & $0.18174(10)$ & $0.45963(12)$ & $0.0126(3)$ \\
\hline H6 & 0.424711 & 0.173024 & 0.531757 & $0.015^{*}$ \\
\hline $\mathrm{C} 5$ & $0.47936(12)$ & $0.11751(10)$ & $0.41315(12)$ & $0.0153(4)$ \\
\hline $\mathrm{H} 5 \mathrm{~A}$ & 0.458726 & 0.060623 & 0.429937 & $0.018^{*}$ \\
\hline H5B & 0.470758 & 0.123374 & 0.341395 & $0.018^{*}$ \\
\hline $\mathrm{C} 4$ & $0.58324(12)$ & $0.12818(10)$ & $0.44703(12)$ & $0.0154(4)$ \\
\hline $\mathrm{C} 3$ & $0.62160(11)$ & $0.21731(10)$ & $0.44544(12)$ & $0.0130(3)$ \\
\hline H3 & 0.623803 & 0.232932 & 0.375406 & $0.016^{*}$ \\
\hline $\mathrm{C} 2$ & $0.55295(11)$ & $0.27918(10)$ & $0.48908(11)$ & $0.0116(3)$ \\
\hline $\mathrm{H} 2$ & 0.550461 & 0.264836 & 0.559444 & $0.014 *$ \\
\hline $\mathrm{C} 13$ & $0.58429(11)$ & $0.36970(10)$ & $0.48314(12)$ & $0.0119(3)$ \\
\hline C14 & $0.60889(11)$ & $0.40364(10)$ & $0.39611(11)$ & $0.0126(3)$ \\
\hline H14 & 0.605537 & 0.370723 & 0.338550 & $0.015^{*}$ \\
\hline $\mathrm{C} 15$ & $0.63859(11)$ & $0.48660(10)$ & $0.39415(12)$ & $0.0135(3)$ \\
\hline $\mathrm{C} 16$ & $0.64548(11)$ & $0.53410(10)$ & $0.47927(12)$ & $0.0124(3)$ \\
\hline $\mathrm{C} 17$ & $0.62075(11)$ & $0.49956(10)$ & $0.56647(11)$ & $0.0124(3)$ \\
\hline $\mathrm{C} 18$ & $0.58935(11)$ & $0.41689(10)$ & $0.56850(12)$ & $0.0124(3)$ \\
\hline $\mathrm{H} 18$ & 0.571624 & 0.393067 & 0.627422 & $0.015^{*}$ \\
\hline C19 & $0.67804(13)$ & $0.47581(11)$ & $0.23027(12)$ & $0.0203(4)$ \\
\hline H19A & 0.618376 & 0.449091 & 0.205160 & $0.031 *$ \\
\hline
\end{tabular}




\begin{tabular}{|c|c|c|c|c|}
\hline H19B & 0.725041 & 0.432818 & 0.250694 & $0.031 *$ \\
\hline $\mathrm{H} 19 \mathrm{C}$ & 0.702184 & 0.510253 & 0.179043 & $0.031 *$ \\
\hline $\mathrm{C} 20$ & $0.76737(14)$ & $0.63474(12)$ & $0.45957(17)$ & $0.0325(5)$ \\
\hline $\mathrm{H} 20 \mathrm{~A}$ & 0.775977 & 0.621427 & 0.391473 & $0.049 *$ \\
\hline $\mathrm{H} 20 \mathrm{~B}$ & 0.810579 & 0.600435 & 0.502496 & $0.049 *$ \\
\hline $\mathrm{H} 20 \mathrm{C}$ & 0.781464 & 0.693938 & 0.471681 & $0.049 *$ \\
\hline $\mathrm{C} 21$ & $0.60812(13)$ & $0.51742(11)$ & $0.73677(12)$ & $0.0197(4)$ \\
\hline $\mathrm{H} 21 \mathrm{~A}$ & 0.543104 & 0.495307 & 0.730657 & $0.030 *$ \\
\hline $\mathrm{H} 21 \mathrm{~B}$ & 0.613472 & 0.561252 & 0.786577 & $0.030 *$ \\
\hline $\mathrm{H} 21 \mathrm{C}$ & 0.652913 & 0.472311 & 0.755689 & $0.030 *$ \\
\hline $\mathrm{C} 22$ & $0.72327(12)$ & $0.22561(11)$ & $0.49349(12)$ & $0.0163(4)$ \\
\hline $\mathrm{H} 22 \mathrm{~A}$ & 0.763183 & 0.181857 & 0.466739 & $0.020^{*}$ \\
\hline H22B & 0.749019 & 0.280445 & 0.475552 & $0.020^{*}$ \\
\hline $\mathrm{C} 23$ & $0.73200(13)$ & $0.21822(12)$ & $0.60461(13)$ & $0.0234(4)$ \\
\hline $\mathrm{H} 23 \mathrm{~A}$ & 0.702708 & 0.164977 & 0.622949 & $0.028 *$ \\
\hline H23B & 0.695760 & 0.264279 & 0.631877 & $0.028 *$ \\
\hline $\mathrm{C} 24$ & $0.83497(14)$ & $0.22117(12)$ & $0.65040(15)$ & $0.0280(4)$ \\
\hline $\mathrm{H} 24 \mathrm{~A}$ & 0.870426 & 0.174045 & 0.624341 & $0.034 *$ \\
\hline H24B & 0.834689 & 0.212755 & 0.721557 & $0.034 *$ \\
\hline $\mathrm{C} 25$ & $0.88844(13)$ & $0.30248(12)$ & $0.63262(15)$ & $0.0259(4)$ \\
\hline $\mathrm{H} 25 \mathrm{~A}$ & 0.955429 & 0.296223 & 0.659602 & $0.031 *$ \\
\hline H25B & 0.888501 & 0.311198 & 0.561497 & $0.031 *$ \\
\hline $\mathrm{C} 26$ & $0.84674(14)$ & $0.37950(12)$ & $0.67733(16)$ & $0.0297(5)$ \\
\hline $\mathrm{H} 26 \mathrm{~A}$ & 0.856737 & 0.375278 & 0.749176 & $0.036^{*}$ \\
\hline H26B & 0.777271 & 0.380692 & 0.659265 & $0.036^{*}$ \\
\hline $\mathrm{C} 27$ & $0.89003(16)$ & $0.46103(13)$ & $0.64518(17)$ & $0.0373(5)$ \\
\hline $\mathrm{H} 27 \mathrm{~A}$ & 0.877272 & 0.465999 & 0.573615 & $0.045^{*}$ \\
\hline H27B & 0.857150 & 0.507928 & 0.674904 & $0.045^{*}$ \\
\hline $\mathrm{C} 28$ & $0.99598(17)$ & $0.47047(15)$ & 0.67097 (19) & $0.0454(6)$ \\
\hline $\mathrm{H} 28 \mathrm{~A}$ & 1.029899 & 0.426339 & 0.637480 & $0.055^{*}$ \\
\hline $\mathrm{H} 28 \mathrm{~B}$ & 1.009964 & 0.462496 & 0.742032 & $0.055^{*}$ \\
\hline $\mathrm{C} 29$ & $1.0331(2)$ & $0.55540(16)$ & $0.6425(2)$ & $0.0583(8)$ \\
\hline H29A & 1.016827 & 0.564801 & 0.572783 & $0.087^{*}$ \\
\hline H29B & 1.102427 & 0.557025 & 0.656495 & $0.087 *$ \\
\hline $\mathrm{H} 29 \mathrm{C}$ & 1.004092 & 0.599083 & 0.679967 & $0.087^{*}$ \\
\hline $\mathrm{C} 32$ & $0.14052(13)$ & $-0.00537(11)$ & $0.55530(14)$ & $0.0227(4)$ \\
\hline $\mathrm{H} 32 \mathrm{~A}$ & 0.181150 & -0.038465 & 0.515784 & $0.034 *$ \\
\hline H32B & 0.089482 & -0.040705 & 0.576454 & $0.034 *$ \\
\hline $\mathrm{H} 32 \mathrm{C}$ & 0.178699 & 0.016486 & 0.612540 & $0.034 *$ \\
\hline $\mathrm{C} 31$ & $-0.02712(14)$ & $0.21502(13)$ & $0.41916(16)$ & $0.0300(5)$ \\
\hline H31A & -0.000302 & 0.271289 & 0.415775 & $0.045^{*}$ \\
\hline H31B & -0.018551 & 0.194756 & 0.486463 & $0.045^{*}$ \\
\hline $\mathrm{H} 31 \mathrm{C}$ & -0.095136 & 0.216619 & 0.397507 & $0.045^{*}$ \\
\hline $\mathrm{C} 30$ & $0.17655(14)$ & $0.31979(13)$ & $0.20387(14)$ & $0.0280(5)$ \\
\hline $\mathrm{H} 30 \mathrm{~A}$ & 0.227213 & 0.291127 & 0.172634 & $0.042 *$ \\
\hline H30B & 0.204707 & 0.361135 & 0.250255 & $0.042 *$ \\
\hline $\mathrm{H} 30 \mathrm{C}$ & 0.134083 & 0.347957 & 0.154131 & $0.042 *$ \\
\hline
\end{tabular}


Atomic displacement parameters $\left(\AA^{2}\right)$

\begin{tabular}{|c|c|c|c|c|c|c|}
\hline & $U^{11}$ & $U^{22}$ & $U^{33}$ & $U^{12}$ & $U^{13}$ & $U^{23}$ \\
\hline C11 & $0.0236(2)$ & $0.0237(2)$ & $0.01052(18)$ & $-0.00088(17)$ & $0.00375(15)$ & $-0.00094(16)$ \\
\hline N1 & $0.0100(7)$ & $0.0096(7)$ & $0.0109(7)$ & $-0.0013(5)$ & $0.0021(5)$ & $0.0001(6)$ \\
\hline $\mathrm{O} 1$ & $0.0126(6)$ & $0.0157(6)$ & $0.0245(6)$ & $-0.0022(5)$ & $0.0034(5)$ & $0.0052(5)$ \\
\hline $\mathrm{O} 2$ & $0.0103(6)$ & $0.0270(7)$ & $0.0234(6)$ & $-0.0016(5)$ & $-0.0009(5)$ & $0.0015(5)$ \\
\hline $\mathrm{O} 3$ & $0.0176(7)$ & $0.0345(8)$ & $0.0227(7)$ & $-0.0059(6)$ & $-0.0058(5)$ & $0.0145(6)$ \\
\hline $\mathrm{O} 4$ & $0.0168(6)$ & $0.0122(6)$ & $0.0437(8)$ & $0.0025(5)$ & $-0.0005(6)$ & $0.0020(6)$ \\
\hline $\mathrm{O5}$ & $0.0217(6)$ & $0.0146(6)$ & $0.0129(6)$ & $-0.0038(5)$ & $0.0037(5)$ & $-0.0035(5)$ \\
\hline O6 & $0.0157(6)$ & $0.0084(6)$ & $0.0217(6)$ & $-0.0021(4)$ & $0.0071(5)$ & $-0.0009(5)$ \\
\hline $\mathrm{O} 7$ & $0.0306(7)$ & $0.0125(6)$ & $0.0134(6)$ & $-0.0001(5)$ & $0.0084(5)$ & $0.0019(5)$ \\
\hline $\mathrm{C} 12$ & $0.0153(8)$ & $0.0159(9)$ & $0.0136(8)$ & $-0.0045(7)$ & $0.0030(6)$ & $-0.0009(7)$ \\
\hline $\mathrm{C} 11$ & $0.0174(9)$ & $0.0193(9)$ & $0.0114(8)$ & $-0.0014(7)$ & $-0.0013(6)$ & $0.0006(7)$ \\
\hline $\mathrm{C} 10$ & $0.0110(8)$ & $0.0177(9)$ & $0.0153(8)$ & $-0.0033(7)$ & $0.0003(6)$ & $-0.0039(7)$ \\
\hline C9 & $0.0150(8)$ & $0.0110(8)$ & $0.0141(8)$ & $-0.0027(6)$ & $0.0038(6)$ & $-0.0033(6)$ \\
\hline $\mathrm{C} 8$ & $0.0148(8)$ & $0.0103(8)$ & $0.0131(8)$ & $0.0002(6)$ & $0.0019(6)$ & $-0.0014(6)$ \\
\hline $\mathrm{C} 7$ & $0.0125(8)$ & $0.0104(8)$ & $0.0123(8)$ & $-0.0001(6)$ & $0.0020(6)$ & $-0.0049(6)$ \\
\hline C6 & $0.0143(8)$ & $0.0103(8)$ & $0.0132(8)$ & $-0.0025(6)$ & $0.0018(6)$ & $0.0011(6)$ \\
\hline $\mathrm{C} 5$ & $0.0159(9)$ & $0.0111(8)$ & $0.0186(8)$ & $-0.0012(7)$ & $0.0006(7)$ & $-0.0022(7)$ \\
\hline $\mathrm{C} 4$ & $0.0144(9)$ & $0.0132(9)$ & $0.0187(8)$ & $0.0001(7)$ & $0.0022(7)$ & $-0.0016(7)$ \\
\hline $\mathrm{C} 3$ & $0.0119(8)$ & $0.0116(8)$ & $0.0154(8)$ & $-0.0005(6)$ & $0.0010(6)$ & $0.0008(6)$ \\
\hline $\mathrm{C} 2$ & $0.0108(8)$ & $0.0126(8)$ & $0.0112(7)$ & $-0.0016(6)$ & $-0.0011(6)$ & $0.0013(6)$ \\
\hline $\mathrm{C} 13$ & $0.0075(8)$ & $0.0119(8)$ & $0.0160(8)$ & $0.0013(6)$ & $-0.0007(6)$ & $0.0019(7)$ \\
\hline $\mathrm{C} 14$ & $0.0139(8)$ & $0.0118(8)$ & $0.0121(8)$ & $0.0008(6)$ & $0.0006(6)$ & $-0.0020(6)$ \\
\hline $\mathrm{C} 15$ & $0.0126(8)$ & $0.0141(8)$ & $0.0139(8)$ & $0.0008(6)$ & $0.0019(6)$ & $0.0023(7)$ \\
\hline C16 & $0.0101(8)$ & $0.0086(8)$ & $0.0185(8)$ & $-0.0004(6)$ & $0.0015(6)$ & $0.0004(6)$ \\
\hline C17 & $0.0097(8)$ & $0.0139(8)$ & $0.0134(8)$ & $0.0012(6)$ & 0.0005 (6) & $-0.0022(7)$ \\
\hline C18 & $0.0104(8)$ & $0.0145(8)$ & $0.0124(8)$ & $-0.0005(6)$ & $0.0013(6)$ & $0.0010(6)$ \\
\hline C19 & $0.0282(10)$ & $0.0210(9)$ & $0.0129(8)$ & $0.0044(8)$ & $0.0076(7)$ & $0.0021(7)$ \\
\hline $\mathrm{C} 20$ & $0.0233(11)$ & $0.0205(10)$ & $0.0565(14)$ & $-0.0085(8)$ & $0.0189(10)$ & $-0.0066(10)$ \\
\hline $\mathrm{C} 21$ & $0.0267(10)$ & $0.0194(9)$ & $0.0133(8)$ & $-0.0008(7)$ & $0.0027(7)$ & $-0.0015(7)$ \\
\hline $\mathrm{C} 22$ & $0.0115(8)$ & $0.0132(8)$ & $0.0239(9)$ & $-0.0013(7)$ & $0.0002(7)$ & $0.0014(7)$ \\
\hline $\mathrm{C} 23$ & $0.0207(10)$ & $0.0235(10)$ & $0.0248(9)$ & $-0.0045(8)$ & $-0.0049(8)$ & $0.0077(8)$ \\
\hline $\mathrm{C} 24$ & $0.0238(10)$ & $0.0252(10)$ & $0.0327(11)$ & $0.0006(8)$ & $-0.0094(8)$ & $0.0039(9)$ \\
\hline $\mathrm{C} 25$ & $0.0148(9)$ & $0.0276(11)$ & 0.0337 (11) & $0.0007(8)$ & $-0.0067(8)$ & $-0.0037(9)$ \\
\hline $\mathrm{C} 26$ & $0.0226(10)$ & $0.0281(11)$ & $0.0372(11)$ & $0.0016(8)$ & $-0.0052(9)$ & $-0.0043(9)$ \\
\hline $\mathrm{C} 27$ & $0.0430(13)$ & $0.0247(11)$ & $0.0427(13)$ & $0.0052(9)$ & $-0.0047(10)$ & $-0.0040(10)$ \\
\hline $\mathrm{C} 28$ & $0.0458(14)$ & $0.0326(13)$ & $0.0557(15)$ & $-0.0129(11)$ & $-0.0076(12)$ & $0.0090(11)$ \\
\hline $\mathrm{C} 29$ & $0.073(2)$ & $0.0385(15)$ & $0.0618(17)$ & $-0.0215(13)$ & $-0.0015(15)$ & $0.0061(13)$ \\
\hline $\mathrm{C} 32$ & $0.0198(10)$ & $0.0145(9)$ & $0.0342(10)$ & $-0.0017(7)$ & $0.0043(8)$ & $0.0080(8)$ \\
\hline $\mathrm{C} 31$ & $0.0163(10)$ & $0.0279(11)$ & $0.0463(12)$ & $0.0036(8)$ & $0.0056(9)$ & $0.0000(9)$ \\
\hline $\mathrm{C} 30$ & $0.0284(11)$ & $0.0326(11)$ & $0.0221(9)$ & $-0.0065(9)$ & $-0.0039(8)$ & $0.0132(9)$ \\
\hline
\end{tabular}

Geometric parameters $(\AA, \stackrel{\circ}{)})$

\begin{tabular}{llll}
\hline $\mathrm{N} 1-\mathrm{C} 6$ & $1.507(2)$ & $\mathrm{C} 18-\mathrm{H} 18$ & 0.9500 \\
$\mathrm{~N} 1-\mathrm{C} 2$ & $1.5092(19)$ & $\mathrm{C} 19-\mathrm{H} 19 \mathrm{~A}$ & 0.9800
\end{tabular}




\begin{tabular}{|c|c|c|c|}
\hline $\mathrm{N} 1-\mathrm{H} 1 \mathrm{~A}$ & $0.92(2)$ & C19-H19B & 0.9800 \\
\hline $\mathrm{N} 1-\mathrm{H} 2 \mathrm{~A}$ & $0.92(2)$ & $\mathrm{C} 19-\mathrm{H} 19 \mathrm{C}$ & 0.9800 \\
\hline $\mathrm{O} 1-\mathrm{C} 9$ & $1.369(2)$ & $\mathrm{C} 20-\mathrm{H} 20 \mathrm{~A}$ & 0.9800 \\
\hline $\mathrm{O} 1-\mathrm{C} 32$ & $1.428(2)$ & $\mathrm{C} 20-\mathrm{H} 20 \mathrm{~B}$ & 0.9800 \\
\hline $\mathrm{O} 2-\mathrm{C} 10$ & $1.3762(19)$ & $\mathrm{C} 20-\mathrm{H} 20 \mathrm{C}$ & 0.9800 \\
\hline $\mathrm{O} 2-\mathrm{C} 31$ & $1.430(2)$ & $\mathrm{C} 21-\mathrm{H} 21 \mathrm{~A}$ & 0.9800 \\
\hline $\mathrm{O} 3-\mathrm{C} 11$ & $1.363(2)$ & $\mathrm{C} 21-\mathrm{H} 21 \mathrm{~B}$ & 0.9800 \\
\hline $\mathrm{O} 3-\mathrm{C} 30$ & $1.427(2)$ & $\mathrm{C} 21-\mathrm{H} 21 \mathrm{C}$ & 0.9800 \\
\hline $\mathrm{O} 4-\mathrm{C} 4$ & $1.212(2)$ & $\mathrm{C} 22-\mathrm{C} 23$ & $1.530(2)$ \\
\hline $\mathrm{O} 5-\mathrm{C} 17$ & $1.3664(19)$ & $\mathrm{C} 22-\mathrm{H} 22 \mathrm{~A}$ & 0.9900 \\
\hline $\mathrm{O} 5-\mathrm{C} 21$ & $1.431(2)$ & $\mathrm{C} 22-\mathrm{H} 22 \mathrm{~B}$ & 0.9900 \\
\hline $\mathrm{O} 6-\mathrm{C} 16$ & $1.3863(19)$ & $\mathrm{C} 23-\mathrm{C} 24$ & $1.533(2)$ \\
\hline $\mathrm{O} 6-\mathrm{C} 20$ & $1.438(2)$ & $\mathrm{C} 23-\mathrm{H} 23 \mathrm{~A}$ & 0.9900 \\
\hline $\mathrm{O} 7-\mathrm{C} 15$ & $1.3691(19)$ & $\mathrm{C} 23-\mathrm{H} 23 \mathrm{~B}$ & 0.9900 \\
\hline $\mathrm{O} 7-\mathrm{C} 19$ & $1.431(2)$ & $\mathrm{C} 24-\mathrm{C} 25$ & $1.535(3)$ \\
\hline $\mathrm{C} 12-\mathrm{C} 11$ & $1.390(2)$ & $\mathrm{C} 24-\mathrm{H} 24 \mathrm{~A}$ & 0.9900 \\
\hline $\mathrm{C} 12-\mathrm{C} 7$ & $1.393(2)$ & $\mathrm{C} 24-\mathrm{H} 24 \mathrm{~B}$ & 0.9900 \\
\hline $\mathrm{C} 12-\mathrm{H} 12$ & 0.9500 & $\mathrm{C} 25-\mathrm{C} 26$ & $1.520(3)$ \\
\hline $\mathrm{C} 11-\mathrm{C} 10$ & $1.394(2)$ & $\mathrm{C} 25-\mathrm{H} 25 \mathrm{~A}$ & 0.9900 \\
\hline $\mathrm{C} 10-\mathrm{C} 9$ & $1.394(2)$ & $\mathrm{C} 25-\mathrm{H} 25 \mathrm{~B}$ & 0.9900 \\
\hline $\mathrm{C} 9-\mathrm{C} 8$ & $1.391(2)$ & $\mathrm{C} 26-\mathrm{C} 27$ & $1.524(3)$ \\
\hline $\mathrm{C} 8-\mathrm{C} 7$ & $1.397(2)$ & $\mathrm{C} 26-\mathrm{H} 26 \mathrm{~A}$ & 0.9900 \\
\hline $\mathrm{C} 8-\mathrm{H} 8$ & 0.9500 & $\mathrm{C} 26-\mathrm{H} 26 \mathrm{~B}$ & 0.9900 \\
\hline $\mathrm{C} 7-\mathrm{C} 6$ & $1.512(2)$ & $\mathrm{C} 27-\mathrm{C} 28$ & $1.513(3)$ \\
\hline $\mathrm{C} 6-\mathrm{C} 5$ & $1.530(2)$ & $\mathrm{C} 27-\mathrm{H} 27 \mathrm{~A}$ & 0.9900 \\
\hline $\mathrm{C} 6-\mathrm{H} 6$ & 1.0000 & $\mathrm{C} 27-\mathrm{H} 27 \mathrm{~B}$ & 0.9900 \\
\hline $\mathrm{C} 5-\mathrm{C} 4$ & $1.508(2)$ & $\mathrm{C} 28-\mathrm{C} 29$ & $1.521(3)$ \\
\hline $\mathrm{C} 5-\mathrm{H} 5 \mathrm{~A}$ & 0.9900 & $\mathrm{C} 28-\mathrm{H} 28 \mathrm{~A}$ & 0.9900 \\
\hline $\mathrm{C} 5-\mathrm{H} 5 \mathrm{~B}$ & 0.9900 & $\mathrm{C} 28-\mathrm{H} 28 \mathrm{~B}$ & 0.9900 \\
\hline $\mathrm{C} 4-\mathrm{C} 3$ & $1.527(2)$ & $\mathrm{C} 29-\mathrm{H} 29 \mathrm{~A}$ & 0.9800 \\
\hline $\mathrm{C} 3-\mathrm{C} 22$ & $1.532(2)$ & $\mathrm{C} 29-\mathrm{H} 29 \mathrm{~B}$ & 0.9800 \\
\hline $\mathrm{C} 3-\mathrm{C} 2$ & $1.544(2)$ & $\mathrm{C} 29-\mathrm{H} 29 \mathrm{C}$ & 0.9800 \\
\hline $\mathrm{C} 3-\mathrm{H} 3$ & 1.0000 & $\mathrm{C} 32-\mathrm{H} 32 \mathrm{~A}$ & 0.9800 \\
\hline $\mathrm{C} 2-\mathrm{C} 13$ & $1.520(2)$ & $\mathrm{C} 32-\mathrm{H} 32 \mathrm{~B}$ & 0.9800 \\
\hline $\mathrm{C} 2-\mathrm{H} 2$ & 1.0000 & $\mathrm{C} 32-\mathrm{H} 32 \mathrm{C}$ & 0.9800 \\
\hline $\mathrm{C} 13-\mathrm{C} 14$ & $1.388(2)$ & $\mathrm{C} 31-\mathrm{H} 31 \mathrm{~A}$ & 0.9800 \\
\hline $\mathrm{C} 13-\mathrm{C} 18$ & $1.395(2)$ & $\mathrm{C} 31-\mathrm{H} 31 \mathrm{~B}$ & 0.9800 \\
\hline $\mathrm{C} 14-\mathrm{C} 15$ & $1.394(2)$ & $\mathrm{C} 31-\mathrm{H} 31 \mathrm{C}$ & 0.9800 \\
\hline C14-H14 & 0.9500 & $\mathrm{C} 30-\mathrm{H} 30 \mathrm{~A}$ & 0.9800 \\
\hline $\mathrm{C} 15-\mathrm{C} 16$ & $1.394(2)$ & $\mathrm{C} 30-\mathrm{H} 30 \mathrm{~B}$ & 0.9800 \\
\hline $\mathrm{C} 16-\mathrm{C} 17$ & $1.395(2)$ & $\mathrm{C} 30-\mathrm{H} 30 \mathrm{C}$ & 0.9800 \\
\hline $\mathrm{C} 17-\mathrm{C} 18$ & $1.397(2)$ & & \\
\hline $\mathrm{C} 6-\mathrm{N} 1-\mathrm{C} 2$ & $110.48(12)$ & $\mathrm{H} 19 \mathrm{~B}-\mathrm{C} 19-\mathrm{H} 19 \mathrm{C}$ & 109.5 \\
\hline $\mathrm{C} 6-\mathrm{N} 1-\mathrm{H} 1 \mathrm{~A}$ & $107.3(13)$ & $\mathrm{O} 6-\mathrm{C} 20-\mathrm{H} 20 \mathrm{~A}$ & 109.5 \\
\hline $\mathrm{C} 2-\mathrm{N} 1-\mathrm{H} 1 \mathrm{~A}$ & $109.8(13)$ & $\mathrm{O} 6-\mathrm{C} 20-\mathrm{H} 20 \mathrm{~B}$ & 109.5 \\
\hline $\mathrm{C} 6-\mathrm{N} 1-\mathrm{H} 2 \mathrm{~A}$ & $110.5(12)$ & $\mathrm{H} 20 \mathrm{~A}-\mathrm{C} 20-\mathrm{H} 20 \mathrm{~B}$ & 109.5 \\
\hline $\mathrm{C} 2-\mathrm{N} 1-\mathrm{H} 2 \mathrm{~A}$ & $106.8(12)$ & $\mathrm{O} 6-\mathrm{C} 20-\mathrm{H} 20 \mathrm{C}$ & 109.5 \\
\hline
\end{tabular}




\begin{tabular}{|c|c|c|c|}
\hline $\mathrm{H} 1 \mathrm{~A}-\mathrm{N} 1-\mathrm{H} 2 \mathrm{~A}$ & $111.9(17)$ & $\mathrm{H} 20 \mathrm{~A}-\mathrm{C} 20-\mathrm{H} 20 \mathrm{C}$ & 109.5 \\
\hline $\mathrm{C} 9-\mathrm{O} 1-\mathrm{C} 32$ & $117.53(13)$ & $\mathrm{H} 20 \mathrm{~B}-\mathrm{C} 20-\mathrm{H} 20 \mathrm{C}$ & 109.5 \\
\hline $\mathrm{C} 10-\mathrm{O} 2-\mathrm{C} 31$ & $111.41(13)$ & $\mathrm{O} 5-\mathrm{C} 21-\mathrm{H} 21 \mathrm{~A}$ & 109.5 \\
\hline $\mathrm{C} 11-\mathrm{O} 3-\mathrm{C} 30$ & $116.83(13)$ & $\mathrm{O} 5-\mathrm{C} 21-\mathrm{H} 21 \mathrm{~B}$ & 109.5 \\
\hline $\mathrm{C} 17-\mathrm{O} 5-\mathrm{C} 21$ & $117.03(13)$ & $\mathrm{H} 21 \mathrm{~A}-\mathrm{C} 21-\mathrm{H} 21 \mathrm{~B}$ & 109.5 \\
\hline $\mathrm{C} 16-\mathrm{O} 6-\mathrm{C} 20$ & $115.55(13)$ & $\mathrm{O} 5-\mathrm{C} 21-\mathrm{H} 21 \mathrm{C}$ & 109.5 \\
\hline $\mathrm{C} 15-\mathrm{O} 7-\mathrm{C} 19$ & $116.26(13)$ & $\mathrm{H} 21 \mathrm{~A}-\mathrm{C} 21-\mathrm{H} 21 \mathrm{C}$ & 109.5 \\
\hline $\mathrm{C} 11-\mathrm{C} 12-\mathrm{C} 7$ & $119.23(15)$ & $\mathrm{H} 21 \mathrm{~B}-\mathrm{C} 21-\mathrm{H} 21 \mathrm{C}$ & 109.5 \\
\hline $\mathrm{C} 11-\mathrm{C} 12-\mathrm{H} 12$ & 120.4 & $\mathrm{C} 23-\mathrm{C} 22-\mathrm{C} 3$ & $114.61(14)$ \\
\hline $\mathrm{C} 7-\mathrm{C} 12-\mathrm{H} 12$ & 120.4 & $\mathrm{C} 23-\mathrm{C} 22-\mathrm{H} 22 \mathrm{~A}$ & 108.6 \\
\hline $\mathrm{O} 3-\mathrm{C} 11-\mathrm{C} 12$ & $124.38(15)$ & $\mathrm{C} 3-\mathrm{C} 22-\mathrm{H} 22 \mathrm{~A}$ & 108.6 \\
\hline $\mathrm{O} 3-\mathrm{C} 11-\mathrm{C} 10$ & $115.27(15)$ & $\mathrm{C} 23-\mathrm{C} 22-\mathrm{H} 22 \mathrm{~B}$ & 108.6 \\
\hline $\mathrm{C} 12-\mathrm{C} 11-\mathrm{C} 10$ & $120.33(15)$ & $\mathrm{C} 3-\mathrm{C} 22-\mathrm{H} 22 \mathrm{~B}$ & 108.6 \\
\hline $\mathrm{O} 2-\mathrm{C} 10-\mathrm{C} 11$ & $119.78(15)$ & $\mathrm{H} 22 \mathrm{~A}-\mathrm{C} 22-\mathrm{H} 22 \mathrm{~B}$ & 107.6 \\
\hline $\mathrm{O} 2-\mathrm{C} 10-\mathrm{C} 9$ & $120.21(15)$ & $\mathrm{C} 22-\mathrm{C} 23-\mathrm{C} 24$ & $113.59(16)$ \\
\hline $\mathrm{C} 11-\mathrm{C} 10-\mathrm{C} 9$ & $120.01(15)$ & $\mathrm{C} 22-\mathrm{C} 23-\mathrm{H} 23 \mathrm{~A}$ & 108.8 \\
\hline $\mathrm{O} 1-\mathrm{C} 9-\mathrm{C} 8$ & $124.86(15)$ & $\mathrm{C} 24-\mathrm{C} 23-\mathrm{H} 23 \mathrm{~A}$ & 108.8 \\
\hline $\mathrm{O} 1-\mathrm{C} 9-\mathrm{C} 10$ & $115.01(14)$ & $\mathrm{C} 22-\mathrm{C} 23-\mathrm{H} 23 \mathrm{~B}$ & 108.8 \\
\hline $\mathrm{C} 8-\mathrm{C} 9-\mathrm{C} 10$ & $120.07(15)$ & $\mathrm{C} 24-\mathrm{C} 23-\mathrm{H} 23 \mathrm{~B}$ & 108.8 \\
\hline $\mathrm{C} 9-\mathrm{C} 8-\mathrm{C} 7$ & $119.35(15)$ & $\mathrm{H} 23 \mathrm{~A}-\mathrm{C} 23-\mathrm{H} 23 \mathrm{~B}$ & 107.7 \\
\hline $\mathrm{C} 9-\mathrm{C} 8-\mathrm{H} 8$ & 120.3 & $\mathrm{C} 23-\mathrm{C} 24-\mathrm{C} 25$ & $114.93(15)$ \\
\hline $\mathrm{C} 7-\mathrm{C} 8-\mathrm{H} 8$ & 120.3 & $\mathrm{C} 23-\mathrm{C} 24-\mathrm{H} 24 \mathrm{~A}$ & 108.5 \\
\hline $\mathrm{C} 12-\mathrm{C} 7-\mathrm{C} 8$ & $120.85(15)$ & $\mathrm{C} 25-\mathrm{C} 24-\mathrm{H} 24 \mathrm{~A}$ & 108.5 \\
\hline $\mathrm{C} 12-\mathrm{C} 7-\mathrm{C} 6$ & $121.59(14)$ & $\mathrm{C} 23-\mathrm{C} 24-\mathrm{H} 24 \mathrm{~B}$ & 108.5 \\
\hline $\mathrm{C} 8-\mathrm{C} 7-\mathrm{C} 6$ & $117.56(14)$ & $\mathrm{C} 25-\mathrm{C} 24-\mathrm{H} 24 \mathrm{~B}$ & 108.5 \\
\hline $\mathrm{N} 1-\mathrm{C} 6-\mathrm{C} 7$ & $111.53(13)$ & $\mathrm{H} 24 \mathrm{~A}-\mathrm{C} 24-\mathrm{H} 24 \mathrm{~B}$ & 107.5 \\
\hline $\mathrm{N} 1-\mathrm{C} 6-\mathrm{C} 5$ & $108.08(13)$ & $\mathrm{C} 26-\mathrm{C} 25-\mathrm{C} 24$ & $114.25(17)$ \\
\hline $\mathrm{C} 7-\mathrm{C} 6-\mathrm{C} 5$ & $113.65(13)$ & $\mathrm{C} 26-\mathrm{C} 25-\mathrm{H} 25 \mathrm{~A}$ & 108.7 \\
\hline $\mathrm{N} 1-\mathrm{C} 6-\mathrm{H} 6$ & 107.8 & $\mathrm{C} 24-\mathrm{C} 25-\mathrm{H} 25 \mathrm{~A}$ & 108.7 \\
\hline $\mathrm{C} 7-\mathrm{C} 6-\mathrm{H} 6$ & 107.8 & $\mathrm{C} 26-\mathrm{C} 25-\mathrm{H} 25 \mathrm{~B}$ & 108.7 \\
\hline $\mathrm{C} 5-\mathrm{C} 6-\mathrm{H} 6$ & 107.8 & $\mathrm{C} 24-\mathrm{C} 25-\mathrm{H} 25 \mathrm{~B}$ & 108.7 \\
\hline $\mathrm{C} 4-\mathrm{C} 5-\mathrm{C} 6$ & $111.89(13)$ & $\mathrm{H} 25 \mathrm{~A}-\mathrm{C} 25-\mathrm{H} 25 \mathrm{~B}$ & 107.6 \\
\hline $\mathrm{C} 4-\mathrm{C} 5-\mathrm{H} 5 \mathrm{~A}$ & 109.2 & $\mathrm{C} 25-\mathrm{C} 26-\mathrm{C} 27$ & $113.49(18)$ \\
\hline $\mathrm{C} 6-\mathrm{C} 5-\mathrm{H} 5 \mathrm{~A}$ & 109.2 & $\mathrm{C} 25-\mathrm{C} 26-\mathrm{H} 26 \mathrm{~A}$ & 108.9 \\
\hline $\mathrm{C} 4-\mathrm{C} 5-\mathrm{H} 5 \mathrm{~B}$ & 109.2 & $\mathrm{C} 27-\mathrm{C} 26-\mathrm{H} 26 \mathrm{~A}$ & 108.9 \\
\hline $\mathrm{C} 6-\mathrm{C} 5-\mathrm{H} 5 \mathrm{~B}$ & 109.2 & $\mathrm{C} 25-\mathrm{C} 26-\mathrm{H} 26 \mathrm{~B}$ & 108.9 \\
\hline $\mathrm{H} 5 \mathrm{~A}-\mathrm{C} 5-\mathrm{H} 5 \mathrm{~B}$ & 107.9 & $\mathrm{C} 27-\mathrm{C} 26-\mathrm{H} 26 \mathrm{~B}$ & 108.9 \\
\hline $\mathrm{O} 4-\mathrm{C} 4-\mathrm{C} 5$ & $121.38(15)$ & $\mathrm{H} 26 \mathrm{~A}-\mathrm{C} 26-\mathrm{H} 26 \mathrm{~B}$ & 107.7 \\
\hline $\mathrm{O} 4-\mathrm{C} 4-\mathrm{C} 3$ & $122.61(15)$ & $\mathrm{C} 28-\mathrm{C} 27-\mathrm{C} 26$ & $115.47(18)$ \\
\hline $\mathrm{C} 5-\mathrm{C} 4-\mathrm{C} 3$ & $115.93(14)$ & $\mathrm{C} 28-\mathrm{C} 27-\mathrm{H} 27 \mathrm{~A}$ & 108.4 \\
\hline $\mathrm{C} 4-\mathrm{C} 3-\mathrm{C} 22$ & $113.23(13)$ & $\mathrm{C} 26-\mathrm{C} 27-\mathrm{H} 27 \mathrm{~A}$ & 108.4 \\
\hline $\mathrm{C} 4-\mathrm{C} 3-\mathrm{C} 2$ & $111.05(13)$ & $\mathrm{C} 28-\mathrm{C} 27-\mathrm{H} 27 \mathrm{~B}$ & 108.4 \\
\hline $\mathrm{C} 22-\mathrm{C} 3-\mathrm{C} 2$ & $111.81(13)$ & $\mathrm{C} 26-\mathrm{C} 27-\mathrm{H} 27 \mathrm{~B}$ & 108.4 \\
\hline $\mathrm{C} 4-\mathrm{C} 3-\mathrm{H} 3$ & 106.8 & $\mathrm{H} 27 \mathrm{~A}-\mathrm{C} 27-\mathrm{H} 27 \mathrm{~B}$ & 107.5 \\
\hline $\mathrm{C} 22-\mathrm{C} 3-\mathrm{H} 3$ & 106.8 & $\mathrm{C} 27-\mathrm{C} 28-\mathrm{C} 29$ & $112.6(2)$ \\
\hline $\mathrm{C} 2-\mathrm{C} 3-\mathrm{H} 3$ & 106.8 & $\mathrm{C} 27-\mathrm{C} 28-\mathrm{H} 28 \mathrm{~A}$ & 109.1 \\
\hline $\mathrm{N} 1-\mathrm{C} 2-\mathrm{C} 13$ & $110.75(12)$ & $\mathrm{C} 29-\mathrm{C} 28-\mathrm{H} 28 \mathrm{~A}$ & 109.1 \\
\hline $\mathrm{N} 1-\mathrm{C} 2-\mathrm{C} 3$ & $109.06(12)$ & $\mathrm{C} 27-\mathrm{C} 28-\mathrm{H} 28 \mathrm{~B}$ & 109.1 \\
\hline
\end{tabular}


$\mathrm{C} 13-\mathrm{C} 2-\mathrm{C} 3$

$\mathrm{N} 1-\mathrm{C} 2-\mathrm{H} 2$

$\mathrm{C} 13-\mathrm{C} 2-\mathrm{H} 2$

$\mathrm{C} 3-\mathrm{C} 2-\mathrm{H} 2$

$\mathrm{C} 14-\mathrm{C} 13-\mathrm{C} 18$

$\mathrm{C} 14-\mathrm{C} 13-\mathrm{C} 2$

$\mathrm{C} 18-\mathrm{C} 13-\mathrm{C} 2$

$\mathrm{C} 13-\mathrm{C} 14-\mathrm{C} 15$

C13-C14-H14

C15-C14- 14

$\mathrm{O} 7-\mathrm{C} 15-\mathrm{C} 16$

$\mathrm{O} 7-\mathrm{C} 15-\mathrm{C} 14$

$\mathrm{C} 16-\mathrm{C} 15-\mathrm{C} 14$

$\mathrm{O} 6-\mathrm{C} 16-\mathrm{C} 15$

$\mathrm{O} 6-\mathrm{C} 16-\mathrm{C} 17$

$\mathrm{C} 15-\mathrm{C} 16-\mathrm{C} 17$

$\mathrm{O} 5-\mathrm{C} 17-\mathrm{C} 16$

$\mathrm{O} 5-\mathrm{C} 17-\mathrm{C} 18$

C16-C17-C18

$\mathrm{C} 13-\mathrm{C} 18-\mathrm{C} 17$

$\mathrm{C} 13-\mathrm{C} 18-\mathrm{H} 18$

C17-C18-H18

O7-C19-H19A

O7- $\mathrm{C} 19-\mathrm{H} 19 \mathrm{~B}$

$\mathrm{H} 19 \mathrm{~A}-\mathrm{C} 19-\mathrm{H} 19 \mathrm{~B}$

O7-C19-H19C

H19A-C19-H19C

$\mathrm{C} 30-\mathrm{O} 3-\mathrm{C} 11-\mathrm{C} 12$

$\mathrm{C} 30-\mathrm{O} 3-\mathrm{C} 11-\mathrm{C} 10$

$\mathrm{C} 7-\mathrm{C} 12-\mathrm{C} 11-\mathrm{O} 3$

$\mathrm{C} 7-\mathrm{C} 12-\mathrm{C} 11-\mathrm{C} 10$

$\mathrm{C} 31-\mathrm{O} 2-\mathrm{C} 10-\mathrm{C} 11$

$\mathrm{C} 31-\mathrm{O} 2-\mathrm{C} 10-\mathrm{C} 9$

$\mathrm{O} 3-\mathrm{C} 11-\mathrm{C} 10-\mathrm{O} 2$

$\mathrm{C} 12-\mathrm{C} 11-\mathrm{C} 10-\mathrm{O} 2$

$\mathrm{O} 3-\mathrm{C} 11-\mathrm{C} 10-\mathrm{C} 9$

$\mathrm{C} 12-\mathrm{C} 11-\mathrm{C} 10-\mathrm{C} 9$

$\mathrm{C} 32-\mathrm{O} 1-\mathrm{C} 9-\mathrm{C} 8$

$\mathrm{C} 32-\mathrm{O} 1-\mathrm{C} 9-\mathrm{C} 10$

$\mathrm{O} 2-\mathrm{C} 10-\mathrm{C} 9-\mathrm{O} 1$

$\mathrm{C} 11-\mathrm{C} 10-\mathrm{C} 9-\mathrm{O} 1$

$\mathrm{O} 2-\mathrm{C} 10-\mathrm{C} 9-\mathrm{C} 8$

$\mathrm{C} 11-\mathrm{C} 10-\mathrm{C} 9-\mathrm{C} 8$

$\mathrm{O} 1-\mathrm{C} 9-\mathrm{C} 8-\mathrm{C} 7$

$\mathrm{C} 10-\mathrm{C} 9-\mathrm{C} 8-\mathrm{C} 7$

$\mathrm{C} 11-\mathrm{C} 12-\mathrm{C} 7-\mathrm{C} 8$

$\mathrm{C} 11-\mathrm{C} 12-\mathrm{C} 7-\mathrm{C} 6$
$113.33(13)$

107.8

107.8

107.8

$121.21(15)$

$121.05(14)$

$117.72(14)$

$119.22(15)$

120.4

120.4

$115.59(14)$

$124.21(14)$

$120.19(15)$

$121.46(14)$

118.14 (14)

$120.27(15)$

$115.47(14)$

$124.74(15)$

$119.79(15)$

$119.32(15)$

120.3

120.3

109.5

109.5

109.5

109.5

109.5

$-3.7(3)$

$175.14(16)$

$177.55(16)$

$-1.2(2)$

$-94.91(19)$

85.34 (19)

$-1.1(2)$

$177.78(15)$

$178.68(15)$

$-2.5(3)$

$-19.5(2)$

$163.47(15)$

0.7 (2)

$-179.09(14)$

$-176.50(14)$

$3.7(2)$

$-178.21(15)$

$-1.3(2)$

$3.6(2)$

$-175.63(15)$
$\mathrm{C} 29-\mathrm{C} 28-\mathrm{H} 28 \mathrm{~B} \quad 109.1$

$\mathrm{H} 28 \mathrm{~A}-\mathrm{C} 28-\mathrm{H} 28 \mathrm{~B} \quad 107.8$

$\mathrm{C} 28-\mathrm{C} 29-\mathrm{H} 29 \mathrm{~A} \quad 109.5$

$\mathrm{C} 28-\mathrm{C} 29-\mathrm{H} 29 \mathrm{~B} \quad 109.5$

$\mathrm{H} 29 \mathrm{~A}-\mathrm{C} 29-\mathrm{H} 29 \mathrm{~B} \quad 109.5$

$\mathrm{C} 28-\mathrm{C} 29-\mathrm{H} 29 \mathrm{C} \quad 109.5$

$\mathrm{H} 29 \mathrm{~A}-\mathrm{C} 29-\mathrm{H} 29 \mathrm{C} \quad 109.5$

$\mathrm{H} 29 \mathrm{~B}-\mathrm{C} 29-\mathrm{H} 29 \mathrm{C} \quad 109.5$

$\mathrm{O} 1-\mathrm{C} 32-\mathrm{H} 32 \mathrm{~A} \quad 109.5$

$\mathrm{O} 1-\mathrm{C} 32-\mathrm{H} 32 \mathrm{~B} \quad 109.5$

$\mathrm{H} 32 \mathrm{~A}-\mathrm{C} 32-\mathrm{H} 32 \mathrm{~B} \quad 109.5$

$\mathrm{O} 1-\mathrm{C} 32-\mathrm{H} 32 \mathrm{C} \quad 109.5$

$\mathrm{H} 32 \mathrm{~A}-\mathrm{C} 32-\mathrm{H} 32 \mathrm{C} \quad 109.5$

$\mathrm{H} 32 \mathrm{~B}-\mathrm{C} 32-\mathrm{H} 32 \mathrm{C} \quad 109.5$

$\mathrm{O} 2-\mathrm{C} 31-\mathrm{H} 31 \mathrm{~A} \quad 109.5$

$\mathrm{O} 2-\mathrm{C} 31-\mathrm{H} 31 \mathrm{~B} \quad 109.5$

$\mathrm{H} 31 \mathrm{~A}-\mathrm{C} 31-\mathrm{H} 31 \mathrm{~B} \quad 109.5$

$\mathrm{O} 2-\mathrm{C} 31-\mathrm{H} 31 \mathrm{C} \quad 109.5$

$\mathrm{H} 31 \mathrm{~A}-\mathrm{C} 31-\mathrm{H} 31 \mathrm{C} \quad 109.5$

$\mathrm{H} 31 \mathrm{~B}-\mathrm{C} 31-\mathrm{H} 31 \mathrm{C} \quad 109.5$

$\mathrm{O} 3-\mathrm{C} 30-\mathrm{H} 30 \mathrm{~A} \quad 109.5$

$\mathrm{O} 3-\mathrm{C} 30-\mathrm{H} 30 \mathrm{~B} \quad 109.5$

$\mathrm{H} 30 \mathrm{~A}-\mathrm{C} 30-\mathrm{H} 30 \mathrm{~B} \quad 109.5$

$\mathrm{O} 3-\mathrm{C} 30-\mathrm{H} 30 \mathrm{C} \quad 109.5$

$\mathrm{H} 30 \mathrm{~A}-\mathrm{C} 30-\mathrm{H} 30 \mathrm{C} \quad 109.5$

$\mathrm{H} 30 \mathrm{~B}-\mathrm{C} 30-\mathrm{H} 30 \mathrm{C} \quad 109.5$

$\mathrm{C} 4-\mathrm{C} 3-\mathrm{C} 2-\mathrm{N} 1 \quad-52.26(17)$

$\mathrm{C} 22-\mathrm{C} 3-\mathrm{C} 2-\mathrm{N} 1 \quad-179.79$ (13)

$\mathrm{C} 4-\mathrm{C} 3-\mathrm{C} 2-\mathrm{C} 13 \quad-176.12(13)$

$\mathrm{C} 22-\mathrm{C} 3-\mathrm{C} 2-\mathrm{C} 13 \quad 56.36(17)$

$\mathrm{N} 1-\mathrm{C} 2-\mathrm{C} 13-\mathrm{C} 14 \quad-72.60(18)$

$\mathrm{C} 3-\mathrm{C} 2-\mathrm{C} 13-\mathrm{C} 14 \quad 50.34$ (19)

$\mathrm{N} 1-\mathrm{C} 2-\mathrm{C} 13-\mathrm{C} 18 \quad 108.71(15)$

$\mathrm{C} 3-\mathrm{C} 2-\mathrm{C} 13-\mathrm{C} 18 \quad-128.36(15)$

$\mathrm{C} 18-\mathrm{C} 13-\mathrm{C} 14-\mathrm{C} 15 \quad-0.4(2)$

$\mathrm{C} 2-\mathrm{C} 13-\mathrm{C} 14-\mathrm{C} 15 \quad-179.07(14)$

$\mathrm{C} 19-\mathrm{O} 7-\mathrm{C} 15-\mathrm{C} 16 \quad 164.77$ (14)

$\mathrm{C} 19-\mathrm{O} 7-\mathrm{C} 15-\mathrm{C} 14 \quad-16.2(2)$

$\mathrm{C} 13-\mathrm{C} 14-\mathrm{C} 15-\mathrm{O} 7 \quad-177.57$ (15)

$\mathrm{C} 13-\mathrm{C} 14-\mathrm{C} 15-\mathrm{C} 16 \quad 1.5$ (2)

$\mathrm{C} 20-\mathrm{O} 6-\mathrm{C} 16-\mathrm{C} 15 \quad-68.8(2)$

$\mathrm{C} 20-\mathrm{O} 6-\mathrm{C} 16-\mathrm{C} 17 \quad 115.49$ (17)

$\mathrm{O} 7-\mathrm{C} 15-\mathrm{C} 16-\mathrm{O} 6 \quad 2.1$ (2)

$\mathrm{C} 14-\mathrm{C} 15-\mathrm{C} 16-\mathrm{O} 6 \quad-177.05$ (14)

$\mathrm{O} 7-\mathrm{C} 15-\mathrm{C} 16-\mathrm{C} 17 \quad 177.72(14)$

$\mathrm{C} 14-\mathrm{C} 15-\mathrm{C} 16-\mathrm{C} 17 \quad-1.4$ (2) 


$\begin{array}{llll}\mathrm{C} 9-\mathrm{C} 8-\mathrm{C} 7-\mathrm{C} 12 & -2.4(2) & \mathrm{C} 21-\mathrm{O} 5-\mathrm{C} 17-\mathrm{C} 16 & -177.99(14) \\ \mathrm{C} 9-\mathrm{C} 8-\mathrm{C} 7-\mathrm{C} 6 & 176.93(14) & \mathrm{C} 21-\mathrm{O}-\mathrm{C} 17-\mathrm{C} 18 & 1.9(2) \\ \mathrm{C} 2-\mathrm{N} 1-\mathrm{C} 6-\mathrm{C} 7 & 169.23(13) & \mathrm{O} 6-\mathrm{C} 16-\mathrm{C} 17-\mathrm{O} 5 & -4.0(2) \\ \mathrm{C} 2-\mathrm{N} 1-\mathrm{C} 6-\mathrm{C} 5 & -65.15(16) & \mathrm{C} 15-\mathrm{C} 16-\mathrm{C} 17-\mathrm{O} 5 & -179.79(14) \\ \mathrm{C} 12-\mathrm{C} 7-\mathrm{C} 6-\mathrm{N} 1 & 28.7(2) & \mathrm{O}-\mathrm{C} 16-\mathrm{C} 17-\mathrm{C} 18 & 176.06(14) \\ \mathrm{C} 8-\mathrm{C} 7-\mathrm{C} 6-\mathrm{N} 1 & -150.55(14) & \mathrm{C} 15-\mathrm{C} 16-\mathrm{C} 17-\mathrm{C} 18 & 0.3(2) \\ \mathrm{C} 12-\mathrm{C} 7-\mathrm{C} 6-\mathrm{C} 5 & -93.73(18) & \mathrm{C} 14-\mathrm{C} 13-\mathrm{C} 18-\mathrm{C} 17 & -0.7(2) \\ \mathrm{C} 8-\mathrm{C} 7-\mathrm{C} 6-\mathrm{C} 5 & 86.98(18) & \mathrm{C} 2-\mathrm{C} 13-\mathrm{C} 18-\mathrm{C} 17 & 178.00(14) \\ \mathrm{N} 1-\mathrm{C} 6-\mathrm{C} 5-\mathrm{C} 4 & 54.93(17) & \mathrm{O} 5-\mathrm{C} 17-\mathrm{C} 18-\mathrm{C} 13 & -179.16(15) \\ \mathrm{C} 7-\mathrm{C} 6-\mathrm{C} 5-\mathrm{C} 4 & 179.29(14) & \mathrm{C} 16-\mathrm{C} 17-\mathrm{C} 18-\mathrm{C} 13 & 0.8(2) \\ \mathrm{C} 6-\mathrm{C} 5-\mathrm{C} 4-\mathrm{O} 4 & 136.04(17) & \mathrm{C} 4-\mathrm{C} 3-\mathrm{C} 22-\mathrm{C} 23 & -72.37(19) \\ \mathrm{C} 6-\mathrm{C} 5-\mathrm{C} 4-\mathrm{C} 3 & -47.10(19) & \mathrm{C} 2-\mathrm{C} 3-\mathrm{C} 22-\mathrm{C} 23 & 53.98(19) \\ \mathrm{O} 4-\mathrm{C} 4-\mathrm{C} 3-\mathrm{C} 22 & -11.0(2) & \mathrm{C} 3-\mathrm{C} 22-\mathrm{C} 23-\mathrm{C} 24 & 176.33(15) \\ \mathrm{C} 5-\mathrm{C} 4-\mathrm{C} 3-\mathrm{C} 22 & 172.14(14) & \mathrm{C} 22-\mathrm{C} 23-\mathrm{C} 24-\mathrm{C} 25 & 61.5(2) \\ \mathrm{O} 4-\mathrm{C} 4-\mathrm{C} 3-\mathrm{C} 2 & -137.80(17) & \mathrm{C} 23-\mathrm{C} 24-\mathrm{C} 25-\mathrm{C} 26 & 63.6(2) \\ \mathrm{C} 5-\mathrm{C} 4-\mathrm{C} 3-\mathrm{C} 2 & 45.39(19) & \mathrm{C} 24-\mathrm{C} 25-\mathrm{C} 26-\mathrm{C} 27 & -170.44(16) \\ \mathrm{C} 6-\mathrm{N} 1-\mathrm{C} 2-\mathrm{C} 13 & -170.33(13) & \mathrm{C} 25-\mathrm{C} 26-\mathrm{C} 27-\mathrm{C} 28 & -60.8(3) \\ \mathrm{C} 6-\mathrm{N} 1-\mathrm{C} 2-\mathrm{C} 3 & 64.30(16) & \mathrm{C} 26-\mathrm{C} 27-\mathrm{C} 28-\mathrm{C} 29 & -176.3(2) \\ & & & \end{array}$

Hydrogen-bond geometry $\left(\AA,{ }^{\circ}\right)$

\begin{tabular}{lllll}
\hline$D-\mathrm{H} \cdots A$ & $D-\mathrm{H}$ & $\mathrm{H} \cdots A$ & $D \cdots A$ & $D-\mathrm{H} \cdots A$ \\
\hline $\mathrm{N} 1-\mathrm{H} 1 A \cdots \mathrm{O}^{6}$ & & \\
$\mathrm{~N} 1-\mathrm{H} 2 A \cdots \mathrm{C} 1$ & $0.92(2)$ & $1.93(2)$ & $2.8500(18)$ & $175.9(19)$ \\
$\mathrm{C} 6-\mathrm{H} 6 \cdots \mathrm{C} 11^{\mathrm{ii}}$ & $0.92(2)$ & $2.18(2)$ & $3.0959(15)$ & $172.6(19)$ \\
$\mathrm{C} 2-\mathrm{H} 2 \cdots \mathrm{C} 1{ }^{i \mathrm{ii}}$ & 1.00 & 2.74 & $3.6526(17)$ & 152 \\
$\mathrm{C} 12-\mathrm{H} 12 \cdots \mathrm{Cl} 1$ & 1.00 & 2.57 & $3.5153(16)$ & 158 \\
$\mathrm{C} 14-\mathrm{H} 14 \cdots \mathrm{C} 11$ & 0.95 & 2.83 & $3.6625(18)$ & 147 \\
$\mathrm{C} 28-\mathrm{H} 28 B \cdots \mathrm{O} 22^{\mathrm{iii}}$ & 0.95 & 2.82 & $3.6144(16)$ & 141 \\
\hline
\end{tabular}

Symmetry codes: (i) $-x+1,-y+1,-z+1$; (ii) $x,-y+1 / 2, z+1 / 2$; (iii) $x+1,-y+1 / 2, z+1 / 2$. 